\title{
The projective limit functor for spectra of webbed spaces
}

\author{
by \\ L. Frerick (Wuppertal), D. KunkLe (Wuppertal) and \\ J. WengenRoth (Trier)
}

\begin{abstract}
We study Palamodov's derived projective limit functor $\operatorname{Proj}^{1}$ for projective spectra consisting of webbed locally convex spaces introduced by Wilde. This class contains almost all locally convex spaces appearing in analysis. We provide a natural characterization for the vanishing of $\operatorname{Proj}^{1}$ which generalizes and unifies results of Palamodov and Retakh for spectra of Fréchet and (LB)-spaces. We thus obtain a general tool for solving surjectivity problems in analysis.
\end{abstract}

1. Introduction. A classical method for solving analytical problems consists in finding local solutions and then searching for corrections which force the local solutions to converge to a global one. For instance, the classical Mittag-Leffler theorem can be proved in that way, and several modern applications of this strategy can be found e.g. in [BMV1, BMV2, DV1, DV2, MTV].

To formalize this method, let us consider a countable projective spectrum $\mathcal{X}$ which consists of linear spaces $X_{n}$ and linear "spectral" maps $\varrho_{n+1}^{n}$ : $X_{n+1} \rightarrow X_{n}$. The "steps" $X_{n}$ might be viewed as the "local parts" of

$$
X=\operatorname{Proj} \mathcal{X}=\left\{\left(x_{n}\right)_{n \in \mathbb{N}} \in \prod_{n \in \mathbb{N}} X_{n}: \varrho_{n+1}^{n} x_{n+1}=x_{n}\right\} .
$$

If $\mathcal{Y}=\left(Y_{n}, \tau_{n+1}^{n}\right)$ is another projective spectrum, $f: \operatorname{Proj} \mathcal{X} \rightarrow \operatorname{Proj} \mathcal{Y}$ is a linear map, and $y \in \operatorname{Proj} \mathcal{Y}$ is given then a local solution of the problem "does $y$ belong to the range of $f$ ?" consists in finding $x_{n} \in X_{n}$ with $f_{n}\left(x_{n}\right)=y_{n}$ (where $f_{n}: X_{n} \rightarrow Y_{n}$ are the local parts of $f$ satisfying $\left.\tau_{n+1}^{n} \circ f_{n+1}=f_{n} \circ \varrho_{n+1}^{n}\right)$. Of course, $\left(x_{n}\right)_{n \in \mathbb{N}}$ need not belong to Proj $\mathcal{X}$, but one may hope to find $r_{n} \in \operatorname{ker} f_{n}$ such that $x=\left(x_{n}-r_{n}\right)_{n \in \mathbb{N}} \in \operatorname{Proj} \mathcal{X}$ and thus $x$ solves $f(x)=y$.

In 1969, V. P. Palamodov introduced the derived functor Proj ${ }^{1}$ which measures the obstacle against the method described above. If $\mathcal{K}$ is the spec-

2000 Mathematics Subject Classification: 46A13, 46M15, 46M18, 46M40.

Key words and phrases: projective limit functor and its derived functor, webbed spaces, Retakh's theorem. 
trum consisting of ker $f_{n}$ then $\operatorname{Proj}^{1} \mathcal{K}=0$ means that the method always works.

This concept is purely algebraic. However, if the spaces $X_{n}$ carry appropriate topologies one can characterize $\operatorname{Proj}^{1}=0$ by topological properties. To mention just one result in this direction, assume that each $X_{n}$ is endowed with a complete metrizable group topology such that the spectral maps are continuous with dense range. Then $\operatorname{Proj}^{1} \mathcal{X}=0$.

In the situation of the classical Mittag-Leffler theorem these assumptions are realized using Runge's theorem. In applications of the $\mathrm{Proj}^{1}$ functor in distribution theory (in particular, when solving PDEs on various spaces of distributions) the steps $X_{n}$ are no longer Fréchet spaces but "dual" to those, e.g. countable inductive limits of Banach spaces (which are called (LB)spaces). For such situations very powerful characterizations of $\operatorname{Proj}^{1}=0$ due to Retakh, Palamodov, Vogt, and the last named author are available.

Yet one step further, if one not only seeks for solvability of PDEs but for a continuous linear solution operator, one can still apply the $\mathrm{Proj}^{1}$ techniques but the structure of the steps is now "LPLB", i.e. an inductive limit of projective limits of (LB)-spaces.

The aim of this article is to unify some results about the vanishing of Proj $^{1}$ known for spectra of Fréchet and (LB)-spaces and extend them to spectra consisting of webbed spaces. This is an extremely wide class of locally convex spaces introduced by M. De Wilde in connection with closed graph theorems. It contains Banach spaces and is stable with respect to countable products, countable direct sums, (closed) subspaces, and (separated) quotients. Thus, all spaces appearing in analysis by repeated applications of countable inductive or projective processes belong to this class.

Here we treat the abstract aspect of this development. Applications to the splitting theory for sequence spaces are contained in the second named author's dissertation $[\mathrm{K}]$.

After finishing the first version of this article we learned that a result very similar to Theorem 3.1 was proved by P. Domański in the unpublished manuscript [D].

2. Projective spectra and webbed spaces. We start by recalling basic definitions and properties of the projective limit functor. As mentioned in the introduction a projective spectrum $\mathcal{X}$ consists of linear spaces $X_{n}$ and linear spectral maps $\varrho_{n+1}^{n}: X_{n+1} \rightarrow X_{n}$. We denote by $\varrho_{k}^{n}=\varrho_{n+1}^{n} \circ \ldots \circ \varrho_{k}^{k-1}$ : $X_{k} \rightarrow X_{n}$ the composition (for $n<k$ ) and by $\varrho_{n}^{n}$ the identity on $X_{n}$. The projective limit is

$$
X=\operatorname{Proj} \mathcal{X}=\left\{\left(x_{n}\right)_{n \in \mathbb{N}} \in \prod_{n \in \mathbb{N}} X_{n}: \varrho_{n+1}^{n} x_{n+1}=x_{n}\right\},
$$


and the canonical map $X \rightarrow X_{n},\left(x_{k}\right)_{k \in \mathbb{N}} \mapsto x_{n}$, is denoted by $\varrho^{n}$. Proj $\mathcal{X}$ is the kernel of the linear map

$$
\Psi: \prod_{n=1}^{\infty} X_{n} \rightarrow \prod_{n=1}^{\infty} X_{n}, \quad\left(x_{n}\right)_{n \in \mathbb{N}} \mapsto\left(\varrho_{n+1}^{n} x_{n+1}-x_{n}\right)_{n \in \mathbb{N}}
$$

and we define the derived functor $\operatorname{Proj}^{1}$ as the cokernel

$$
\operatorname{Proj}^{1} \mathcal{X}=\prod_{n=1}^{\infty} X_{n} / \operatorname{im} \Psi \text {. }
$$

This formula was obtained by Palamodov as a characterization of his definition of the derived functor in terms of homological algebra (see [P1, P2, $\mathrm{V} 1, \mathrm{~V} 2, \mathrm{~W} 3]$ ). Instead of going into details we just mention a simple result which is used in applications (as explained in the introduction).

Proposition 2.1. Let $\mathcal{X}, \mathcal{Y}$, and $\mathcal{Z}$ be projective spectra and

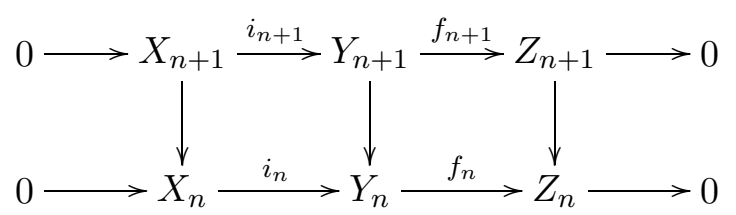

commutative diagrams (the vertical arrows are the spectral maps) with exact rows (i.e. $f_{n}$ is surjective and $i_{n}$ is the embedding of $X_{n}=\operatorname{ker} f_{n}$ into $Y_{n}$ ). Then $\operatorname{Proj}^{1} \mathcal{X}=0$ implies that the induced map $f: \operatorname{Proj} \mathcal{Y} \rightarrow \operatorname{Proj} \mathcal{Z}$ is surjective.

Proof. Given $z=\left(z_{n}\right)_{n \in \mathbb{N}} \in \operatorname{Proj} \mathcal{Z}$ there are $y_{n} \in Y_{n}$ with $z_{n}=f_{n} y_{n}$. Using the commutativity we get $y_{n}-\varrho_{n+1}^{n} y_{n+1} \in \operatorname{ker} f_{n}$. Hence there are $x_{n} \in X_{n}$ with $y_{n}-\varrho_{n+1}^{n} y_{n+1}=i_{n} x_{n}$, and $\operatorname{Proj}^{1} \mathcal{X}=0$ implies the existence of "corrections" $r_{n} \in X_{n}$ with $x_{n}=r_{n}-\varrho_{n+1}^{n} r_{n+1}$. Then $\widetilde{y}_{n}=y_{n}-i_{n} r_{n}$ defines an element $\widetilde{y} \in \operatorname{Proj} \mathcal{Y}$ with $f \widetilde{y}=z$.

The proposition above is in a certain sense even a characterization: If the conclusion holds for all such commutative diagrams then $\operatorname{Proj}^{1} \mathcal{X}=0$. Using this one can show by elementary calculations that $\operatorname{Proj}^{1} \mathcal{X}=0$ if and only if $\operatorname{Proj}^{1} \widetilde{\mathcal{X}}=0$ where $\widetilde{\mathcal{X}}$ is some subsequence of $\mathcal{X}$, i.e. for some increasing sequence $k(n)$ we have $\widetilde{X}_{n}=X_{k(n)}$ and $\widetilde{\varrho}_{n+1}^{n}=\varrho_{k(n+1)}^{k(n)}$.

Let us state one of the fundamental results about $\operatorname{Proj}^{1}=0$ which is due to Retakh $[\mathrm{R}]$ (the implications $(1) \Rightarrow(2) \Rightarrow(3)$ ), Palamodov $[\mathrm{P} 2]((3) \Rightarrow(1))$ and the last named author [W2] $((4) \Rightarrow(1))$. We denote by $\mathcal{B D}(X)$ the system of Banach discs in a locally convex space $X$, i.e. bounded absolutely convex sets $B$ such that the linear span $[B]$ endowed with $\|x\|_{B}=\inf \{\lambda>0: x \in$ $\lambda B\}$ is a Banach space. 
Theorem 2.2. Let $\mathcal{X}=\left(X_{n}, \varrho_{n+1}^{n}\right)$ be a projective spectrum of (LB)spaces and continuous linear spectral maps. The following conditions are equivalent:

(1) $\operatorname{Proj}^{1} \mathcal{X}=0$.

(2) There are $B_{n} \in \mathcal{B D}\left(X_{n}\right)$ such that

( $\alpha) \varrho_{n+1}^{n}\left(B_{n+1}\right) \subseteq B_{n}$,

( $\beta) \forall n \in \mathbb{N} \exists m \geq n \forall k \geq m \varrho_{m}^{n}\left(X_{m}\right) \subseteq \varrho_{k}^{n}\left(X_{k}\right)+B_{n}$.

(3) There are $B_{n} \in \mathcal{B D}\left(X_{n}\right)$ such that

$(\alpha) \varrho_{n+1}^{n}\left(B_{n+1}\right) \subseteq B_{n}$,

$(\widetilde{\beta}) \forall n \in \mathbb{N} \exists m \geq n \varrho_{m}^{n}\left(X_{m}\right) \subseteq \varrho^{n}(\operatorname{Proj} \mathcal{X})+B_{n}$.

(4) There are $D_{n} \in \mathcal{B D}\left(X_{n}\right)$ such that

$$
\forall n \in \mathbb{N} \exists m \geq n \quad \varrho_{m}^{n}\left(X_{m}\right) \subseteq \varrho^{n}(\operatorname{Proj} \mathcal{X})+D_{n} .
$$

If in the theorem the spaces are in addition Montel spaces and the spectral maps have dense range then one can drop condition $(\beta)$ in $(2)$; this is proved in [W1]. More results of that type are in [BV, FW] and all this can also be found in [W3].

Now we will recall the definition of webbed spaces (as given e.g. in $[\mathrm{J}]$ ), the class of spaces to which we want to generalize (at least parts of) the theorem above.

Definition 2.3. Let $X$ be a locally convex space. A web in $X$ is a family $\left(C_{\alpha_{1}, \ldots, \alpha_{k}}\right)_{k, \alpha_{1}, \ldots, \alpha_{k} \in \mathbb{N}}$ of absolutely convex sets with the following properties:

(1) $\bigcup_{\alpha=1}^{\infty} C_{\alpha}=X$.

(2) $\bigcup_{n=1}^{\infty} C_{\alpha_{1}, \ldots, \alpha_{k}, n}=C_{\alpha_{1}, \ldots, \alpha_{k}}$ for all $k, \alpha_{1}, \ldots, \alpha_{k} \in \mathbb{N}$.

(3) For every $\left(\alpha_{k}\right)_{k \in \mathbb{N}} \in \mathbb{N}^{\mathbb{N}}$ there is $\left(\lambda_{k}\right)_{k \in \mathbb{N}} \in(0, \infty)^{\mathbb{N}}$ such that for every sequence $\left(x_{k}\right)_{k \in \mathbb{N}}$ in $X$ with $x_{k} \in C_{\alpha_{1}, \ldots, \alpha_{k}}$ the series $\sum_{k=1}^{\infty} \lambda_{k} x_{k}$ converges in $X$.

The web is called strict if in (3) we additionally have, for every $k_{0} \in \mathbb{N}$,

$$
\sum_{k=k_{0}}^{\infty} \lambda_{k} x_{k} \in C_{\alpha_{1}, \ldots, \alpha_{k_{0}}} .
$$

The web is called ordered if $\alpha_{k} \leq \beta_{k}, k \in \mathbb{N}$, implies $C_{\alpha_{1}, \ldots, \alpha_{k}} \subseteq C_{\beta_{1}, \ldots, \beta_{k}}$.

For a sequence $\left(\alpha_{k}\right)_{k \in \mathbb{N}}$ and $j, k \in \mathbb{N}$ we will write $C_{\alpha \mid j, k}:=C_{\alpha_{j}, \ldots, \alpha_{k}}$ and $C_{\alpha \mid k}:=C_{\alpha_{1}, \ldots, \alpha_{k}}$.

The class of spaces having a (strict or ordered) web is closed with respect to sequentially closed subspaces, separated quotients, countable products and countable direct sums. Thus also countable inductive and projective limits of webbed spaces are webbed. We will now show that the sequence $\left(\lambda_{k}\right)_{k \in \mathbb{N}}$ in (3) can always be taken independently of $\alpha$ as $\lambda_{k}=2^{-(k+1)}$. 
Lemma 2.4. Let $\mathcal{C}=\left(C_{\alpha \mid k}\right)_{k, \alpha_{1}, \ldots, \alpha_{k} \in \mathbb{N}}$ be a (strict) web in the locally convex space $X$. For $\alpha=\left(\alpha_{k}\right)_{k \in \mathbb{N}} \in \mathbb{N}^{\mathbb{N}}$ and $\left(x_{k}\right)_{k \in \mathbb{N}} \in \prod_{k \in \mathbb{N}} C_{\alpha \mid k}$ the series $\sum_{k=p}^{\infty} 2^{-(k+1)} x_{k}$ converges in $X$ (to an element of $2^{1-p} C_{\alpha \mid p}$ ).

Proof. For simplicity we assume $p=1$ (the proof for general $p \in \mathbb{N}$ is the same).

For a given sequence $\beta=\left(\beta_{k}\right)_{k \in \mathbb{N}}$ of natural numbers, we take a sequence $\left(\lambda_{k}\right)_{k \in \mathbb{N}}$ as in (3) and we find a strictly increasing sequence $\left(l_{k}\right)_{k \in \mathbb{N}}$ of natural numbers such that for every $k \in \mathbb{N}$ we have

$$
l_{k-1} \geq k \quad \text { and } \quad \sum_{\nu=l_{k}+1}^{\infty} 2^{-\nu} \leq \lambda_{k+1} .
$$

Now for a sequence $\left(x_{k}\right)_{k \in \mathbb{N}}$ in $X$ such that $x_{k} \in C_{\alpha \mid k}$ for every $k \in \mathbb{N}$, we define a sequence $\left(y_{k}\right)_{k \in \mathbb{N}}$ by

$$
y_{1}:=0 \quad \text { and } \quad y_{k}:=\lambda_{k}^{-1} \sum_{\nu=l_{k-1}+1}^{l_{k}} 2^{-\nu} x_{\nu} \quad \text { for } k \geq 2 .
$$

For $k \geq 2$ and $l_{k-1}<\nu \leq l_{k}$ we have $x_{\nu} \in C_{\alpha \mid \nu} \subseteq C_{\alpha \mid l_{k-1}} \subseteq C_{\alpha \mid k}$, thus absolute convexity and $\sum_{\nu=l_{k}+1}^{l_{k+1}} 2^{-\nu} \leq \lambda_{k+1}$ imply $y_{k} \in C_{\alpha \mid k}$. According to the choice of $\left(\lambda_{k}\right)_{k \in \mathbb{N}}$, the series $\sum_{k=2}^{\infty} \lambda_{k} y_{k}$ converges (to an element $\left.z \in C_{\alpha \mid 2}\right)$ and thus also the sequence

$$
\sum_{\nu=1}^{l_{k}} 2^{-(\nu+1)} x_{\nu}=2^{-1} \sum_{\nu=1}^{l_{1}} 2^{-\nu} x_{\nu}+2^{-1} \sum_{j=1}^{k} \lambda_{j} y_{j}
$$

must converge as $k \rightarrow \infty$ (to an element $w \in 2^{-1} C_{\alpha \mid 1}+2^{-1} C_{\alpha \mid 2} \subseteq C_{\alpha \mid 1}$ ).

Choose for every $n \in \mathbb{N}$ the maximal $k(n) \in \mathbb{N}$ such that $l_{k(n)}<n$. If we show that the sequence $\left(r_{n}\right)_{n \in \mathbb{N}}$ defined by

$$
r_{n}:=\sum_{\nu=l_{k(n)}+1}^{n} 2^{-(\nu+1)} x_{\nu} \in \lambda_{k(n)+1} C_{\alpha \mid k(n)+1}
$$

converges to zero, the proof will be finished. For this it suffices to show that for each subsequence $n(m)$ there is a further subsequence $m(i)$ such that $r_{n(m(i))}$ tends to 0 . But if $m(i)$ is chosen such that $k(n(m(i)))$ is strictly increasing, even the series $\sum_{i=1}^{\infty} r_{n(m(i))}$ converges. This shows $r_{n} \rightarrow 0$.

3. The Retakh-Palamodov theorem for webbed spaces. In all results of this section about projective spectra of locally convex spaces it is tacitly assumed that the spectral maps are continuous with respect to the given topologies on the step spaces. We start with a natural generalization of the equivalence of (1), (2), and (3) in Theorem 2.2. 
TheOREm 3.1. Let $\mathcal{X}=\left(X_{n}, \varrho_{n+1}^{n}\right)_{n \in \mathbb{N}}$ be a projective spectrum of locally convex spaces $X_{n}$ having ordered webs $\mathcal{C}^{n}=\left(C_{\alpha \mid k}^{n}\right)_{k, \alpha_{1}, \ldots, \alpha_{k} \in \mathbb{N}}$. The following conditions are equivalent:

(1) $\operatorname{Proj}^{1} \mathcal{X}=0$.

(2) There is $\left(\alpha_{\nu}\right)_{\nu \in \mathbb{N}} \in \mathbb{N}^{\mathbb{N}}$ such that for all $n \in \mathbb{N}$ there exists $m \geq n$ such that for all $k \geq m$ we have

$$
\varrho_{m}^{n} X_{m} \subseteq \varrho_{k}^{n} X_{k}+\bigcap_{j=1}^{n}\left(\varrho_{n}^{j}\right)^{-1}\left(C_{\alpha \mid j, n}^{j}\right) .
$$

If every web $\mathcal{C}^{n}, n \in \mathbb{N}$, is strict, then (1) and (2) are equivalent to

(3) There is a sequence $\left(\alpha_{\nu}\right)_{\nu \in \mathbb{N}} \in \mathbb{N}^{\mathbb{N}}$ such that for all $n \in \mathbb{N}$ there exists $m \geq n$ with

$$
\varrho_{m}^{n} X_{m} \subseteq \varrho^{n} \operatorname{Proj} \mathcal{X}+\bigcap_{j=1}^{n}\left(\varrho_{n}^{j}\right)^{-1}\left(C_{\alpha \mid j, n}^{j}\right) .
$$

Proof. We first show that (1) implies (2). If we consider $\Pi:=\prod_{n=1}^{\infty} X_{n}$ with the topology $\sigma$ defined by the basis of neighbourhoods of zero

$$
U_{n}:=\prod_{j=1}^{n}\{0\} \times \prod_{j=n+1}^{\infty} X_{j}, \quad n \in \mathbb{N}
$$

(i.e. $\sigma$ is the product of the discrete topologies), then $(\Pi, \sigma)$ is complete and metrizable, thus it is Baire. Since $X_{1}=\bigcup_{j=1}^{\infty} C_{j}^{1}$ and since the mapping $\Psi: \Pi \rightarrow \Pi$ defined in the previous section is surjective, there exists $\alpha_{1} \in \mathbb{N}$ such that $\Psi\left(C_{\alpha_{1}}^{1} \times \prod_{j=2}^{\infty} X_{j}\right)$ is not meagre in $\Pi$. Since $\mathcal{C}^{1}$ and $\mathcal{C}^{2}$ are ordered, it follows that

$$
C_{\alpha_{1}}^{1} \times X_{2}=\bigcup_{n=1}^{\infty} C_{\alpha_{1}, n}^{1} \times C_{n}^{2}
$$

and we find $\alpha_{2} \in \mathbb{N}$ such that $\Psi\left(C_{\alpha_{1}, \alpha_{2}}^{1} \times C_{\alpha_{2}}^{2} \times \prod_{j=3}^{\infty} X_{j}\right)$ is not meagre in $\Pi$. Inductively we can find a sequence $\left(\alpha_{\nu}\right)_{\nu \in \mathbb{N}}$ such that for all $n \in \mathbb{N}$ the set

$$
A_{n}:=\Psi\left(\prod_{j=1}^{n} C_{\alpha \mid j, n}^{j} \times \prod_{j=n+1}^{\infty} X_{j}\right)
$$

is not meagre in $\Pi$, which implies that every $\bar{A}_{n}^{\sigma}$ contains interior points. Also the origin is an interior point (as $\bar{A}_{n}^{\sigma}$ is absolutely convex and $\sigma$ has a basis of the zero-neighbourhood filter consisting of vector spaces), which implies that for all $n \in \mathbb{N}$ there exists $m \geq n$ such that for all $k \geq m$,

$$
\prod_{j=1}^{m-1}\{0\} \times \prod_{j=m}^{\infty} X_{j} \subseteq A_{n}+\prod_{j=1}^{k-1}\{0\} \times \prod_{j=k}^{\infty} X_{j} .
$$


This implies that for given $x \in X_{m}$ and $k \geq m$ there is a sequence $\left(z_{j}\right)_{j \in \mathbb{N}} \in$ $\prod_{j=1}^{n} C_{\alpha \mid j, n}^{j} \times \prod_{j=n+1}^{\infty} X_{j}$ such that

$$
\left(\delta_{j m} x\right)_{j \in \mathbb{N}}-\Psi\left(\left(z_{j}\right)_{j \in \mathbb{N}}\right) \in \prod_{j=1}^{k-1}\{0\} \times \prod_{j=k}^{\infty} X_{j} .
$$

From this we get $z_{j}-\varrho_{j+1}^{j} z_{j+1}=0$ for $j \leq k-1, j \neq m$, and $z_{m}-\varrho_{m+1}^{m} z_{m+1}$ $=x$, whence

$$
\varrho_{m}^{n} x=\varrho_{m}^{n} z_{m}-\varrho_{m+1}^{n} z_{m+1}=z_{n}+\varrho_{k}^{n} z_{k} \in \bigcap_{j=1}^{n}\left(\varrho_{n}^{j}\right)^{-1}\left(C_{\alpha \mid j, n}^{j}\right)+\varrho_{k}^{n} X_{k},
$$

so the inclusion in (2) is proved.

In the proof that (2) implies (1) we may assume without loss of generality that the inclusion in (2) holds with $m(n)=n+1$ (because of the remark after Proposition 2.1). Take a sequence $\left(x_{n}\right)_{n \in \mathbb{N}} \in \prod_{n=1}^{\infty} X_{n}$. To find a sequence $\left(w_{n}\right)_{n \in \mathbb{N}}$ with $\Psi\left(\left(w_{n}\right)_{n \in \mathbb{N}}\right)=\left(x_{n}\right)_{n \in \mathbb{N}}$, we set $y_{1}:=y_{2}:=0$, and find inductively

$$
y_{n} \in X_{n} \quad \text { and } \quad z_{n} \in 2^{-(n+1)} \bigcap_{j=1}^{n}\left(\varrho_{n}^{j}\right)^{-1}\left(C_{\alpha \mid j, n}^{j}\right)
$$

such that

$$
\varrho_{n+1}^{n}\left(y_{n+1}-x_{n+1}\right)=\varrho_{n+2}^{n}\left(y_{n+2}\right)+z_{n} \quad \text { for all } n \in \mathbb{N} .
$$

As for $k \geq n$ we know that $\varrho_{k}^{n} z_{k} \in 2^{-(k+1)} C_{\alpha \mid n, k}^{n}$, Lemma 2.4 implies the convergence of $\sum_{k=n}^{\infty} \varrho_{k}^{n} z_{k}:=r_{n}$. We have $r_{n}-\varrho_{n+1}^{n} r_{n+1}=z_{n}$, so if we set

$$
w_{n}:=\varrho_{n+1}^{n} y_{n+1}-r_{n}+x_{n},
$$

for all $n \in \mathbb{N}$ we get

$w_{n}-\varrho_{n+1}^{n} w_{n+1}=\varrho_{n+1}^{n}\left(y_{n+1}-x_{n+1}\right)-\varrho_{n+2}^{n} y_{n+2}-\left(r_{n}-\varrho_{n+1}^{n} r_{n+1}\right)+x_{n}=x_{n}$.

It is obvious that (3) implies (2) (as $\left.\varrho^{n}(\operatorname{Proj} \mathcal{X}) \subseteq \varrho_{k}^{n} X_{k}\right)$, so it remains to show that (1) implies (3). We construct a sequence $\left(\alpha_{\nu}\right) \in \mathbb{N}^{\mathbb{N}}$ as in the first part of this proof and endow $\Pi$ with the topology $\mathcal{S}$ having the 0-neighbourhood basis

$$
\left\{\varepsilon \prod_{j=1}^{n} C_{\alpha \mid j, n}^{j} \times \prod_{j>n} X_{n}: \varepsilon>0, n \in \mathbb{N}\right\} .
$$

The strictness of the webs implies that $(\Pi, \mathcal{S})$ is again a complete metrizable group. Now, $\Psi:(\Pi, \mathcal{S}) \rightarrow(\Pi, \sigma)$ has closed graph and is almost open (by the choice of $\alpha_{\nu}$ ), i.e. images of 0 -neighbourhoods are not meagre. The Schauder lemma (see e.g. [MV, Lemma 3.9]) implies that $\Psi$ is open, and as before this yields the inclusion required in (3). 
Let us mention that the theorem above has been used in [W3, Theorem 3.3.3] to answer a question of Palamodov [P2, §12.2] about topological consequences of $\operatorname{Proj}^{1} \mathcal{X}=0$ :

If $\mathcal{X}$ is a projective spectrum of locally convex spaces having strict ordered webs then $\operatorname{Proj}^{1} \mathcal{X}=0$ implies $\operatorname{Proj}^{+} \mathcal{X}=0$, i.e. $\Psi: \prod_{n \in \mathbb{N}} X_{n}$ $\rightarrow \prod_{n \in \mathbb{N}} X_{n}$ is open.

On the other hand, there are spectra $\mathcal{X}$ of complete locally convex spaces such that $\operatorname{Proj}^{1} \mathcal{X}=0$ but $\Psi$ is not open [W3, Example 3.3.2].

In the proof of the theorem (which is quite similar to Retakh's proof $[\mathrm{R}]$ for spectra of (LB)-spaces) we did not use all properties of webs, but particularly the following:

Definition 3.2. Let $X$ be a locally convex space, $\left(A_{r}\right)_{r \in \mathbb{N}}$ a decreasing sequence of subsets of $X$. We call $\left(A_{r}\right)_{r \in \mathbb{N}}$ completing (respectively strict) if there exists a sequence $\left(\lambda_{r}\right)_{r \in \mathbb{N}} \in(0, \infty)^{\mathbb{N}}$ such that for all $\left(x_{r}\right)_{r \in \mathbb{N}} \in$ $\prod_{r=1}^{\infty} A_{r}$ and all $p \in \mathbb{N}$ the series $\sum_{r=p}^{\infty} \lambda_{r} x_{r}$ converges in $X$ (to an element in $A_{p}$ ).

The same proof as in Lemma 2.4 shows that for a completing or strict sequence $\left(A_{r}\right)_{r \in \mathbb{N}}$ we can always choose $\lambda_{r}=2^{-(r+1)}, r \in \mathbb{N}$. As in Theorem 3.1 we have:

Proposition 3.3. Let $\mathcal{X}=\left(X_{n}, \varrho_{n+1}^{n}\right)_{n \in \mathbb{N}}$ be a projective spectrum of locally convex spaces $X_{n}$ having completing sequences $\left(A_{r}^{n}\right)_{r \in \mathbb{N}}$ with $\varrho_{n+1}^{n} A_{r}^{n+1}$ $\subseteq A_{r}^{n}$ for all $n, r \in \mathbb{N}$. Then $\operatorname{Proj}^{1} \mathcal{X}=0$ if for all $n \in \mathbb{N}$ there is $m \geq n$ such that for all $k \geq m$ we have

$$
\varrho_{m}^{n} X_{m} \subseteq \varrho_{k}^{n} X_{k}+A_{n}^{n}
$$

REMARK. To verify the assumptions of the proposition, it is sufficient to show

$$
\varrho_{n+1}^{n} X_{n+1} \subseteq \varrho_{n+2}^{n} X_{n+2}+A_{n}^{n}
$$

for all $n \in \mathbb{N}$, since we can repeatedly use it to get the condition for all $k \geq m=n+1$.

To get a sufficient condition for $\operatorname{Proj}^{1} \mathcal{X}=0$ in terms of the websets $C_{\alpha \mid k}^{n}$ and without the intersection appearing in Theorem 3.1 we need simple permanence properties of strict sequences:

Lemma 3.4. Let $f: X \rightarrow Y$ be a continuous linear map between locally convex spaces. If $\left(A_{r}\right)_{r \in \mathbb{N}}$ and $\left(B_{r}\right)_{r \in \mathbb{N}}$ are strict sequences in $X$ respectively $Y$ then $\left(f\left(A_{r}\right)+B_{r}\right)_{r \in \mathbb{N}}$ and $\left(A_{r} \cap f^{-1}\left(B_{r}\right)\right)_{r \in \mathbb{N}}$ are again strict. 
Proof. If $x_{r}=f\left(a_{r}\right)+b_{r}$ with $a_{r} \in A_{r}$ and $b_{r} \in B_{r}, r \in \mathbb{N}$, then for all $p \in \mathbb{N}$ we have

$$
\sum_{r=p}^{\infty} 2^{-(r+1)} a_{r} \in A_{p} \quad \text { and } \quad \sum_{r=p}^{\infty} 2^{-(r+1)} b_{r} \in B_{p}
$$

(in particular, the series converge), thus we get the convergence of

$$
\sum_{r=p}^{\infty} 2^{-(r+1)} x_{r} \in f\left(A_{p}\right)+B_{p}
$$

In the same way, if $x_{r} \in A_{r} \cap f^{-1}\left(B_{r}\right), r \in \mathbb{N}$, then

$$
\sum_{r=p}^{\infty} 2^{-(r+1)} x_{r} \in A_{p} \quad \text { and } \quad \sum_{r=p}^{\infty} 2^{-(r+1)} f\left(x_{r}\right) \in B_{p}, \quad p \in \mathbb{N}
$$

imply that

$$
\sum_{r=p}^{\infty} 2^{-(r+1)} x_{r} \in A_{p} \cap f^{-1}\left(B_{p}\right), \quad p \in \mathbb{N} .
$$

We now prove a sophisticated "webbed version" of the sufficiency of the condition

$$
\begin{aligned}
\forall n \in \mathbb{N} \exists m \geq n \forall k \geq m \exists B_{n} & \in \mathcal{B D}\left(X_{n}\right) \\
& \forall B_{m} \in \mathcal{B D}\left(X_{m}\right) \exists B_{k} \in \mathcal{B D}\left(X_{k}\right) \quad \varrho_{m}^{n} B_{m} \subseteq \varrho_{k}^{n} B_{k}+B_{n}
\end{aligned}
$$

for $\operatorname{Proj}^{1} \mathcal{X}=0$. This is an improved formulation of a result proved in [FW] and $[\mathrm{BV}]$ recently observed by $\mathrm{M}$. Langenbruch (personal communication).

TheOREM 3.5. Let $\mathcal{X}=\left(X_{n}, \varrho_{n+1}^{n}\right)_{n \in \mathbb{N}}$ be a projective spectrum of locally convex spaces $X_{n}$ having strict ordered webs $\mathcal{C}^{n}=\left(C_{\alpha \mid k}^{n}\right)_{k, \alpha_{1}, \ldots, \alpha_{k} \in \mathbb{N}}$ with

$$
2 C_{\alpha_{1}, \ldots, \alpha_{r}}^{n} \subseteq C_{2 \alpha_{1}, \ldots, 2 \alpha_{r}}^{n} \quad \text { for all } \alpha \in \mathbb{N}^{\mathbb{N}} .
$$

Then the following condition implies $\operatorname{Proj}^{1} \mathcal{X}=0$ :

$$
\begin{aligned}
\forall n \in \mathbb{N} \exists m \geq n \forall k \geq m \exists \alpha & \in \mathbb{N}^{\mathbb{N}} \forall \beta \in \mathbb{N}^{\mathbb{N}} \exists \gamma \in \mathbb{N}^{\mathbb{N}} \\
\forall r & \in \mathbb{N} \exists s \in \mathbb{N} \forall t \in \mathbb{N} \quad \varrho_{m}^{n} C_{\beta \mid s}^{m} \subseteq \varrho_{k}^{n} C_{\gamma \mid t}^{k}+C_{\alpha \mid r}^{n} .
\end{aligned}
$$

Proof. Without loss of generality we assume $m(n)=n+1$ (see the remark after Proposition 2.1). Because of $(*)$, we have with the same quantifiers as in the statement of the theorem

$$
\varrho_{n+1}^{n} C_{\beta \mid s}^{n+1} \subseteq \varrho_{k}^{n} C_{\gamma \mid t}^{k}+2^{-1} C_{\alpha \mid r}^{n} .
$$

We will show that this implies

$$
\varrho_{n+1}^{n} X_{n} \in \varrho_{n+2}^{n} X_{n+2}+A_{n}^{n} .
$$


Using the assumption, we choose $\alpha^{1}$ according to $n=1, m=2$ and $k=3$ and $\alpha^{2}$ according to $n=2, m=3$ and $k=4$. Then there is a sequence $\gamma=\left(\gamma_{r}\right)_{r \in \mathbb{N}}$ and a strictly increasing sequence $(s(r))_{r \in \mathbb{N}}$ such that for all $t, r \in \mathbb{N}$,

$$
\varrho_{2}^{1} C_{\alpha^{2} \mid s(r)}^{2} \subseteq \varrho_{3}^{1} C_{\gamma \mid t}^{3}+C_{\alpha^{1} \mid r}^{1} .
$$

We define, for all $r, t \in \mathbb{N}$,

$$
A_{r}^{1}:=C_{\alpha^{1} \mid r}^{1}, \quad B_{r, t}:=\left(\varrho_{2}^{1}\right)^{-1}\left(A_{r}^{1}\right) \cap\left(\varrho_{3}^{2}\left(C_{\gamma \mid t}^{3}\right)+C_{\alpha^{2} \mid s(r)}^{2}\right), \quad A_{r}^{2}:=B_{r, r} .
$$

Then (1) implies $C_{\alpha^{2} \mid s(r)}^{2} \subseteq \varrho_{3}^{2}\left(C_{\gamma \mid t}^{3}\right)+\left(\varrho_{2}^{1}\right)^{-1}\left(A_{r}^{1}\right)$ and therefore also

$$
C_{\alpha^{2} \mid s(r)}^{2} \subseteq \varrho_{3}^{2}\left(C_{\gamma \mid t}^{3}\right)+B_{r, t}
$$

for all $r, t \in \mathbb{N}$. The choice of $\alpha^{2}$ implies the existence of a sequence $\delta=$ $\left(\delta_{r}\right)_{r \in \mathbb{N}}$ and of a strictly increasing sequence $(\widetilde{s}(r))_{r \in \mathbb{N}}$ such that for all $r, \widetilde{t} \in \mathbb{N}$,

$$
\varrho_{3}^{2} C_{\gamma \mid \widetilde{s}(r)}^{3} \subseteq \varrho_{4}^{2}\left(C_{\delta \mid \widetilde{t}}^{4}\right)+2^{-1} C_{\alpha^{2} \mid r}^{2} .
$$

Inserting $s(r)$ instead of $r$ into the last inclusion and defining $\widehat{s}(r):=\widetilde{s}(s(r))$, we deduce from (2), for all $r, t, \widetilde{t} \in \mathbb{N}$,

$$
\varrho_{3}^{2} C_{\gamma \mid \widehat{s}(r)}^{3} \subseteq \varrho_{4}^{2}\left(C_{\delta \mid \tilde{t}}^{4}\right)+2^{-1} B_{r, t}+2^{-1} \varrho_{3}^{2} C_{\gamma \mid t}^{3} .
$$

This inclusion for $t=\widehat{s}(r+1)$ gives us, for all $r, \tilde{t} \in \mathbb{N}$,

$$
\varrho_{3}^{2} C_{\gamma \mid \widehat{s}(r)}^{3} \subseteq \varrho_{4}^{2}\left(C_{\delta \mid \widetilde{t}}^{4}\right)+2^{-1} A_{r}^{2}+2^{-1} \varrho_{3}^{2} C_{\gamma \mid \widehat{s}(r+1)}^{3} .
$$

Here we have also used the fact that $(\widehat{s}(r))_{r \in \mathbb{N}}$ is strictly increasing and the sets $B_{r, t}$ are ordered by inclusion with respect to $t$, so

$$
B_{r, \widehat{s}(r+1)} \subseteq B_{r, r}=A_{r}^{2} .
$$

We show that this implies

$$
\varrho_{3}^{2} C_{\gamma \mid \widehat{s}(r)}^{3} \subseteq 4 \varrho_{4}^{2}\left(C_{\delta \mid \tau}^{4}\right)+2 A_{r}^{2}
$$

for all $r, \tau \in \mathbb{N}$. For this we take fixed $r, \tau \in \mathbb{N}$ and $x_{0} \in C_{\gamma \mid \widehat{s}(r)}^{3}$. Then (4) applied to $\tilde{t}=\tau$ gives

$$
y_{0} \in C_{\delta \mid \tau}^{4}, \quad z_{0} \in A_{r}^{2}, \quad x_{1} \in C_{\gamma \mid \widehat{s}(r+1)}^{3}
$$

such that

$$
\varrho_{3}^{2} x_{0}=\varrho_{4}^{2} y_{0}+2^{-1} z_{0}+2^{-1} \varrho_{3}^{2} x_{1} .
$$

For every $p \in \mathbb{N}$ we get inductively using (4) for $\widetilde{t}=\tau+p$ elements

$$
y_{p} \in C_{\delta \mid \tau+p}^{4}, \quad z_{p} \in A_{r+p}^{2}, \quad x_{p+1} \in C_{\gamma \mid \widehat{s}(r+p+1)}^{3}
$$

such that

$$
\varrho_{3}^{2} x_{p}=\varrho_{4}^{2} y_{p}+2^{-1} z_{p}+2^{-1} \varrho_{3}^{2} x_{p+1},
$$


thus

$$
\varrho_{3}^{2} x_{0}=\varrho_{4}^{2} \sum_{p=0}^{q} 2^{-p} y_{p}+\sum_{p=0}^{q} 2^{-(p+1)} z_{p}+\varrho_{3}^{2}\left(2^{-(q+1)} x_{q+1}\right)
$$

for all $q \in \mathbb{N}$. Now the web $\mathcal{C}^{4}$ is strict and $\left(A_{r}^{2}\right)_{r \in \mathbb{N}}$ is strict because of Lemma 3.4. Thus by 2.4 the two series converge to elements in $4 C_{\delta \mid \tau}^{4}$ and $2 A_{r}^{2}$ respectively, so the right hand side of the last equation converges to an element in $4 \varrho_{4}^{2} C_{\delta \mid \tau}^{4}+2 A_{r}^{2}$, which proves (5).

The next step is to show that for all sequences $\beta=\left(\beta_{r}\right)_{r \in \mathbb{N}}$ there is a sequence $\sigma=\left(\sigma_{r}\right)_{r \in \mathbb{N}}$ and a sequence $(\bar{s}(r))_{r \in \mathbb{N}}$ such that for all $\tau, r \in \mathbb{N}$ we have

$$
\varrho_{3}^{2} C_{\beta \mid \bar{s}(r)}^{3} \subseteq \varrho_{4}^{2} C_{\sigma \mid \tau}^{k}+A_{r}^{2} .
$$

Fix $\beta$; then according to the choice of $\alpha^{2}$ there are sequences $\eta, s^{\prime} \in \mathbb{N}^{\mathbb{N}}$ such that for all $r, \widetilde{\tau} \in \mathbb{N}$,

$$
\varrho_{3}^{2} C_{\beta \mid s^{\prime}(r)}^{3} \subseteq \varrho_{4}^{2} C_{\eta \mid \widetilde{\tau}}^{4}+C_{\alpha^{2} \mid r}^{2}
$$

and so, defining $\bar{s}(r):=s^{\prime}(s(r))$, we get, for all $r, t, \widetilde{\tau} \in \mathbb{N}$,

$$
\varrho_{3}^{2} C_{\beta \mid \bar{s}(r)}^{3} \subseteq \varrho_{4}^{2} C_{\eta \mid \widetilde{\tau}}^{4}+C_{\alpha_{2} \mid s(r)}^{2} \subseteq \varrho_{4}^{2} C_{\eta \mid \widetilde{\tau}}^{4}+\varrho_{3}^{2} C_{\gamma \mid t}^{3}+B_{r, t}
$$

where in the last inclusion we have used (2). Inserting $t=\widehat{s}(r)$ and using (5) we get, according to the definition of $A_{r}^{2}$,

$$
\begin{aligned}
\varrho_{3}^{2} C_{\beta \mid \bar{s}(r)}^{3} & \subseteq \varrho_{4}^{2} C_{\eta \mid \widetilde{\tau}}^{4}+\varrho_{3}^{2} C_{\gamma \mid \widehat{s}(r)}^{3}+A_{r}^{2} \subseteq \varrho_{4}^{2} C_{\eta \mid \widetilde{\tau}}^{4}+4 \varrho_{4}^{2} C_{\delta \mid \tau}^{4}+2 A_{r}^{2}+A_{r}^{2} \\
& \subseteq \varrho_{4}^{2} C_{\eta \mid \widetilde{\tau}}^{4}+4 \varrho_{4}^{2} C_{\delta \mid \tau}^{4}+3 A_{r}^{2}
\end{aligned}
$$

for all $r, \widetilde{\tau}, \tau \in \mathbb{N}$. Putting $\widetilde{\tau}=\tau$ and defining $\sigma_{k}=\max \left\{\eta_{k}, \delta_{k}\right\}, k \in \mathbb{N}$, we conclude

$$
\varrho_{3}^{2} C_{\beta \mid \bar{s}(r)}^{3} \subseteq 5 \varrho_{4}^{2} C_{\sigma \mid \tau}^{4}+3 A_{r}^{2}
$$

Using this inclusion for $8 \beta$ instead of $\beta$ and taking into account $(*)$, we get the inclusion (6).

So far, starting from $\left(A_{r}^{1}\right)_{r \in \mathbb{N}}$, we have constructed a strict sequence $\left(A_{r}^{2}\right)_{r \in \mathbb{N}}$ of subsets satisfying (6) and $\varrho_{2}^{1} A_{r}^{2} \subseteq A_{r}^{1}$ for all $r \in \mathbb{N}$. Proceeding in the same way with $A_{r}^{n-1}$ instead of $A_{r}^{1}$ we can construct inductively strict sequences $\left(A_{r}^{n}\right)_{r \in \mathbb{N}}, n \in \mathbb{N}$, that satisfy $\varrho_{n+1}^{n} A_{r}^{n+1} \subseteq A_{r}^{n}$ and for all $\beta \in \mathbb{N}^{\mathbb{N}}$ there exists $\sigma \in \mathbb{N}^{\mathbb{N}}$ such that for all $r \in \mathbb{N}$ there exists $s(r) \in \mathbb{N}$ such that for all $t \in \mathbb{N}$,

$$
\varrho_{n+1}^{n} C_{\beta \mid s(r)}^{n+1} \subseteq \varrho_{n+2}^{n} C_{\sigma \mid t}^{n+2}+A_{r}^{n}
$$

To finally arrive at $\operatorname{Proj}^{1} \mathcal{X}=0$, we use the Remark after Proposition 3.3: 
If $n \in \mathbb{N}$ and $x \in X_{n+1}$ are given, there is a sequence $\beta$ of natural numbers such that $x \in C_{\beta \mid s}^{n+1}$ for all $s \in \mathbb{N}$. Thus (7) with $r=n$ implies

$$
\varrho_{n+1}^{n} x \in \varrho_{n+2}^{n} X_{n+2}+A_{n}^{n}
$$

so we have proved the inclusion in Proposition 3.3, which implies $\operatorname{Proj}^{1} \mathcal{X}$ $=0$.

\section{References}

[BMV1] R. W. Braun, R. Meise, and D. Vogt, Applications of the projective limit functor to convolution and partial differential equations, in: Advances in the Theory of Fréchet Spaces, T. Terzioğlu (ed.), NATO Adv. Sci. Inst. Ser. C Math. Phys. Sci. 287, Kluwer, Dordrecht, 1989, 29-46.

[BMV2] - - - - Existence of fundamental solutions and surjectivity of convolution operators on classes of ultradifferentiable functions, Proc. London Math. Soc. 61 (1990), 344-370.

[BV] R. W. Braun and D. Vogt, A sufficient condition for $\operatorname{Proj}^{1} \mathcal{X}=0$, Michigan Math. J. 44 (1997), 149-156.

[D] P. Domański, On the splitting theory for the space of distributions, unpublished manuscript.

[DV1] P. Domański and D. Vogt, A splitting theory for the space of distributions, Studia Math. 140 (2000), 57-77.

[DV2] - - Distributional complexes split for positive dimensions, J. Reine Angew. Math. 522 (2000), 63-79.

[DV3] - - - A splitting theorem for the space of smooth functions J. Funct. Anal. 153 (1998), 203-248.

[FW] L. Frerick and J. Wengenroth, A sufficient condition for the vanishing of the derived projective limit functor, Arch. Math. (Basel) 67 (1996), 296-301.

[J] H. Jarchow, Locally Convex Spaces, Teubner, Stuttgart, 1981.

[K] D. Kunkle, Splitting of power series spaces of PLS-type, dissertation, Wuppertal, 2001.

[MV] R. Meise and D. Vogt, Introduction to Functional Analysis, Clarendon Press, New York, 1997.

[MTV] R. Meise, B. A. Taylor, and D. Vogt, Characterisation of the linear partial differential operators with constant coefficients that admit a continuous linear right inverse, Ann. Inst. Fourier (Grenoble) 40 (1990), 619-655.

[P1] V. P. Palamodov, The projective limit functor in the category of linear topological spaces, Mat. Sb. 75 (1968), 567-603 (in Russian); English transl.: Math. USSR-Sb. 4 (1968), 529-558.

[P2] - Homological methods in the theory of locally convex spaces, Uspekhi Mat. Nauk 26 (1971), no. 1, 3-66 (in Russian); English transl.: Russian Math. Surveys 26 (1971), no. 1, 1-64.

[R] V. S. Retakh, Subspaces of a countable inductive limit, Dokl. Akad. Nauk SSSR 194 (1970), 1277-1279 (in Russian); English transl.: Soviet Math. Dokl. 11 (1970), 1384-1386.

[V1] D. Vogt, Lectures on projective spectra of (DF)-spaces, seminar lectures, AG Funktionalanalysis Düsseldorf, Wuppertal, 1987. 
[V2] D. Vogt, Topics on projective spectra of LB-spaces, in: Advances in the Theory of Fréchet Spaces, T. Terzioğlu (ed.), NATO Adv. Sci. Inst. Ser. C Math. Phys. Sci. 287, Kluwer, Dordrecht, 1989, 11-27.

[W1] J. Wengenroth, Inductive spectra of Fréchet spaces, Studia Math. 120 (1996), $247-258$.

[W2] - A new characterization of $\operatorname{Proj}^{1} \mathcal{X}$ for countable spectra of (LB)-spaces, Proc. Amer. Math. Soc. 127 (1999), 737-744.

[W3] - Derived Functors in Functional Analysis, Lecture Notes in Math., Springer, to appear.

Fachbereich Mathematik

Bergische Universität - GH Wuppertal

D-42097 Wuppertal, Germany

E-mail: frerick@math.uni-wuppertal.de

Current address of D. Kunkle:

Theoretische Informatik I

FernUniversität Hagen

D-58084 Hagen, Germany

E-mail: daren.kunkle@fernuni-hagen.de
Fachbereich IV - Mathematik

Universität Trier D-54286 Trier, Germany E-mail: wengen@uni-trier.de

Received March 25, 2002

Revised version December 19, 2002 